\title{
Avaliação do impacto da qualidade de energia elétrica na produção industrial: proposta de metodologia
}

\author{
Miguel Otávio Barreto Campelo de Melo \\ Programa de Pós-Graduação em Engenharia de Produção - UFPB \\ $\underline{\text { mobcmelo@ct.ufpb.br }}$
}

\begin{abstract}
A Qualidade da Energia Elétrica Industrial vem sendo recentemente objeto de diversas pesquisas no setor. Os seus indicadores atualmente são definidos mediante patamares e índices tradicionais de avaliação e não estão incluídos conceitos e procedimentos a partir de um ponto de vista global dos sistemas elétricos que levem em consideração os impactos econômicos que a perda da Qualidade da Energia Elétrica causará no mercado industrial regional. Diante do exposto, verifica-se que a metodologia atualmente utilizada necessita de aperfeiçoamentos para uma melhor tomada de decisão na sua gestão. Este artigo tem por objetivo propor uma nova "Metodologia de Avaliação da Qualidade de Energia Elétrica na Produção Industrial" com novos indicadores técnicos e econômicos que levem em consideração os custos associados à perda desta qualidade. Com a finalidade de validar a metodologia proposta, foram realizadas pesquisas em indústrias no Nordeste do Brasil nos estados da Paraíba, Pernambuco, Alagoas, Sergipe e Bahia.
\end{abstract}

Palavras-Chave: custo da qualidade de energia; economia industrial; gestão industrial

Power Quality is a field of studies that has recently been object of concern and research.. Power Quality indicators of the supply of Electric Energy used nowadays are defined by means of traditional ratings of evaluation and do not included concepts and procedures from a global perspective of the electric systems that take into account the economic impacts of the loss of the Quality of the Electric power in the regional industrial areas. Thus, one verifies that nowadays the present methodology needs improvement for better decision-making in its management. The aim of the present work is to propose a new "Evaluation Methodology of the Electric Power Quality in the Industrial Production" with the new technical and economic indicators that take in consideration the costs associated to the loss of this quality. Some researches were accomplished in some industries in Brazil's Northeast, in the states of Paraíba, Pernambuco, Alagoas, Sergipe and Bahia.

Keywords: power quality cost; industrial economy; industrial management

\section{Introdução}

A qualidade do fornecimento de energia elétrica tem ocupado cada vez mais importância tanto para as distribuidoras quanto para os consumidores . Essa preocupação tem se tornado mais evidente devido a diversos fatores, entre os quais pode-se enumerar os seguintes:

\section{... O uso cada vez mais freqüente nas indús- trias de equipamentos eletronicamente controla- dos e processos automatizados. Entretanto, tais dispositivos são extremamente sensíveis e neces- sitam de energia com mais qualidade e com peque- nas flutuações de freqüência e tensão;}

... Alguns eventos que no passado ocorriam

\begin{abstract}
nos sistemas de energia elétrica de potência e que não causavam efeitos, atualmente resultam em desligamentos dos equipamentos. Pode-se citar os eventos devidos a problemas de indução e de compatibilidade eletromagnética nas subestações entre outros.
\end{abstract}

No Brasil, o problema de Qualidade da Energia Elétrica é semelhante ao de outros países como os EUA, e tem sido agravado nos últimos anos à medida que as indústrias, em geral, têm investido em automatização de seus sistemas produtivos, na busca de obter uma melhoria nos seus processos a fim de enfrentar a concorrência devido à globalização da economia. 
Ressalta-se que mesmo nos países industrializados se observa a necessidade de se rever os critérios de qualidade de energia e de confiabilidade. Esses tópicos também são de grande importância no atual contexto de mudanças em que passa o setor elétrico brasileiro, onde a presença do estado está cada vez menor e, conseqüentemente, ocorre uma maior atuação do setor privado. Neste contexto, a oferta de energia com boa qualidade é um dos fatores de competição entre as próprias concessionárias (ALVES, 1999; HEYDT, 1995; GARCIA, 1999).

A determinação dos custos das perdas devido à qualidade da energia elétrica vêm sendo pesquisados e contabilizados nos últimos vinte anos, com trabalhos realizados em vários locais do mundo, destacando-se os de Billinton e Wacker (1989), no Canadá; Kariuki e Allan (1996), na Inglaterra; e pelo Electric Power Research Institute (EPRI), nos Estados Unidos, Palo Alto-Califórnia, cuja síntese foi publicada por Sullivan (1996), onde são apresentados alguns resultados para variações de curta duração, além dos desligamentos devido à interrupção de energia. McGranaghan e Roettger (2002), nos EUA, apresentam uma metodologia para estimar os custos dos afundamentos a partir dos prejuízos decorrentes de um desligamento de curta duração, atribuindo percentuais que variam de acordo com a sua magnitude. No Brasil, merecem destaque as pesquisas sobre os custos das interrupções realizadas por Magalhães (2001) em São Paulo, e na Região Nordeste do Brasil, por Leitão (2002), sendo que diversos trabalhos da literatura também abordam este tema (BILLINTON, 1989; DIALYNAS, 1998; PEREIRA, 1998).

Os indicadores de qualidade do fornecimento da energia elétrica no Brasil utilizados atualmente são definidos mediante patamares e índices de interrupção denominados "continuidade", ou de "flutuação de energia" denominados "conformidade". Entretanto, não são incluídos conceitos e procedimentos de Qualidade de Energia Elétrica a partir de um ponto de vista global dos sistemas elétricos que levem em consideração os impactos econômicos que a perda de qualidade da energia elétrica causará no mercado industrial regional. Verifica-se, portanto, que a metodologia atualmente utilizada para avaliação ainda necessita de aperfeiçoamento com dados e indicadores adicionais que levem em consideração tanto os aspectos econômicos associados, bem como as características e peculiaridades das empresas industriais situadas em uma determinada região.

Por outro lado, verifica-se que diversas concessionárias de energia e indústrias consumidoras já estão implantando no planejamento empresarial o seu "Programa de Qualidade de Energia Elétrica", principalmente porque estes valores influem nos custos das tarifas a serem cobradas pelas distribuidoras.

\section{A energia elétrica, qualidade e definições}

A energia chega às indústrias e residências por meio de um complexo que é denominado de Sistemas de Potência de Energia, composto de usinas geradoras e subestações, linhas de transmissão, linhas de distribuição e alimentadores. O sistema de energia começa nas usinas geradoras, onde a energia elétrica é gerada, em seguida é transportada por uma complexa rede de Linhas de Transmissão Aéreas ou de Cabos subterrâneos até alcançar seus centros consumidores.

No entanto, para manter o nível de tensão dentro de certos limites operacionais aceitáveis, tanto em nível de transmissão como de distribuição, são necessárias medidas de controle pois os sistemas de distribuição/transmissão estão constantemente sujeitos a ocasionais variações de tensão, que mesmo dentro de limites pré-estabelecidos, podem causar operações incorretas de sensíveis equipamentos elétricos nos diversos setores (OLESKOVICZ, 2006).

Para avaliar o quanto um sistema está operando fora de suas condições normais, duas grandezas elétricas básicas podem ser empregadas. São elas: a tensão e a freqüência. A freqüência em um sistema interligado situa-se na faixa de $60 \mathrm{~Hz} \pm 0,5 \mathrm{~Hz}$. Por outro lado, em relação à tensão, três aspectos principais devem ser observados:

1-Forma de onda, que deve ser o mais próximo possível de uma "Senóide";

2-Simetria do Sistema Elétrico e

3- Magnitudes das tensões dentro de limites aceitáveis.

Entretanto, existem alguns fenômenos, aleatórios ou intrínsecos, que ocorrem no sistema elétrico fazendo com que os aspectos acima citados sofram alterações, deteriorando a qualidade do fornecimento de energia elétrica.

Na última década, há uma evolução da participação do setor industrial na economia do país atingindo cerca de $40 \%$ do PIB (IBGE, 2005). Na Figura 1, é apresentada a evolução do consumo de energia elétrica no Brasil dos diversos setores no período 2001-2004, onde se observa um acentuado crescimento do setor industrial.

Na Região do Nordeste do Brasil, segundo Cavalcanti (2002), os cenários para 2020 são otimistas, a participação do PIB industrial dessa Região que teve uma evolução de $18 \%$ em 1970 a $28 \%$ em 1998, tem perspectivas de atingir o patamar de $30 \%$. 


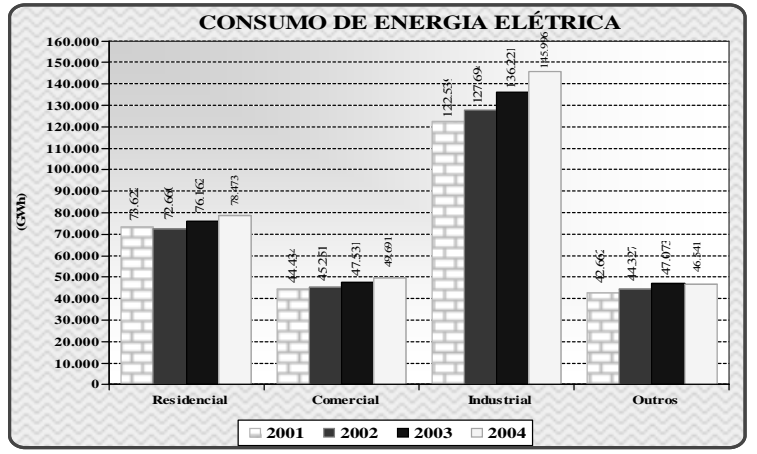

Figura 1 - Evolução do Consumo de Energia Elétrica dos Diversos Setores (Período 2001-2004).

\subsection{A qualidade de energia elétrica: introdução}

Qualidade de Energia Elétrica designa uma área de estudos pertinente aos Sistemas de Potência de Energia Elétrica. Este tema vem sendo, em anos recentes, objeto de preocupação e de esforços coordenados de diagnósticos e de análise para que se atinja o maior entendimento possível sobre o mesmo. Estes conceitos têm sido nas últimas três décadas, objetos de estudos e pesquisas (BRONZEADO, 1997).

Em um sistema elétrico trifásico ideal, as tensões em qualquer ponto deveriam ser, de forma permanente, perfeitamente senoidais, equilibradas, e com amplitude e freqüência constantes. Qualquer desvio, acima de certos limites, na característica desses parâmetros é considerado um problema de qualidade de energia (BONATTO, 1999). Na Figura 2, é apresentado o gráfico de amplitude de tensão ao longo tempo, resultando numa senoidal típica de um sistema ideal onde 1 (um) ciclo corresponde a 16 milisegundos.

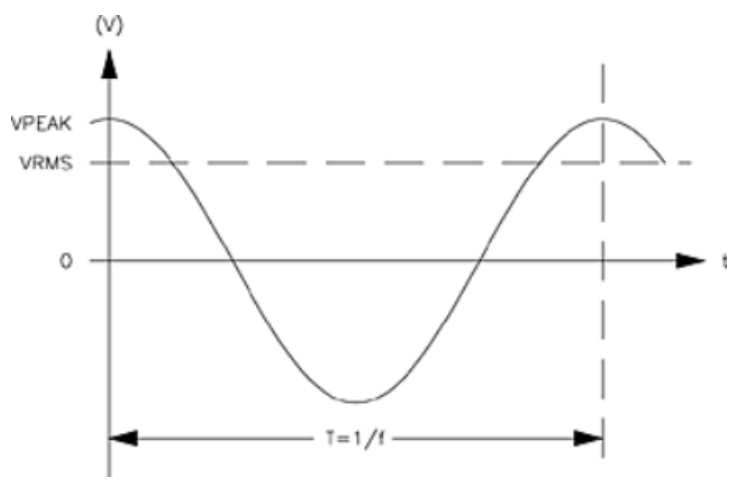

Figura 2 - Gráfico da Onda Senoidal Típica da Tensão Fonte: Bronzeado (1997).

Na última década, têm aumentado consideravelmente o número de equipamentos, aparelhos e controles sensíveis à qualidade de energia, como computadores, controles de processo, e equipamentos de comunicação, cujos sistemas são mais sensíveis à qualidade da energia do que as tradicionais cargas anteriormente existentes (HEYDT,1998).

\subsection{Qualidade de energia elétrica: grandezas}

... Variações sustentadas de tensão: são variações de valor eficaz da tensão entre dois níveis consecutivos, com duração incerta, porém maior que ou igual a 1 (um) minuto;

... Variações momentâneas de tensão: são variações momentâneas no valor eficaz da tensão entre dois níveis consecutivos, com duração incerta, porém menor do que 1 minuto. As Variações Momentâneas de Tensão podem ser classificadas como: Interrupções Momentâneas de Tensão (IMT): Elevações Momentâneas de Tensão (EMT): e Afundamento Momentâneo de Tensão (AMT): (AMANTEGUI, 1998; BOLLEN, 1998; STECIUK, 1996);

... V Variações instantâneas de tensão: são variações súbitas do valor instantâneo da tensão. São subdivididas em: Cortes na Tensão, Transitórios Oscilatórios de Tensão e Surtos de Tensão;

... Variações sustentadas ou momentâneas de freqüência: são pequenos desvios do valor da freqüência fundamental das tensões decorrentes do desequilíbrio entre a geração da energia elétrica e a demanda solicitada pela carga;

... Distúrbios quase-permanentes: são distúrbios causados pela operação de cargas não-lineares. São definidos como: Flutuação de Tensão; Desequilíbrio de Tensão; Distorção Harmônica Total e Cintilação.

A Figura 3 apresenta, para efeito ilustrativo, os gráficos das formas de onda da energia elétrica dos principais distúrbios de Qualidade de Energia Elétrica em um sistema de Potência de Energia Elétrica (BRONZEADO et al., 1997).

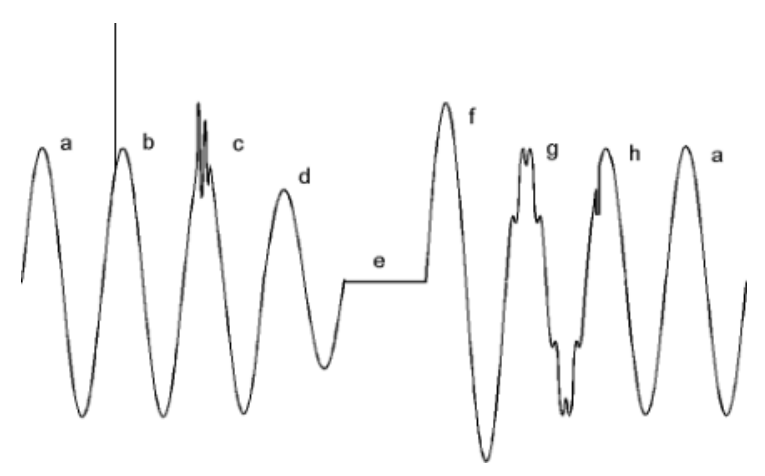

Figura 3 - Distúrbios Associados à Qualidade de Energia Elétrica

Fonte: Bronzeado et al.(1997).

As formas de onda são de acordo com a seguinte legenda: a) Tensão normal; b) Surto de Tensão; c) Transitório 
Oscilatório de Tensão; d) Subtensão Momentânea; e) Interrupção Momentânea de Tensão; f) Sobretensão Momentânea; g) Distorção Harmônica; h) Cortes na Tensão.

\subsection{Qualidade de energia elétrica: indices de avaliação}

A seguir, é apresentada a descrição dos índices tradicionais de qualidade de energia elétrica atualmente usados pelas concessionárias e consumidores do setor elétrico. (IEEE PES, 1995a; IEEE PES, 1995b; GOVERNODESÃO PAULO, 1997).

1-Variações Sustentadas de Freqüência $(\Delta \mathrm{Fs})$

2-Variações Momentâneas de Freqüência $(\Delta \mathrm{Fm})$

3-Variações Sustentadas de Tensão

3.1 Índices de Continuidade

Freqüência Equivalente de Interrupção (FREQ)

Duração Equivalente de Interrupção (DREQ)

Energia Interrompida (ENES)

Freqüência de Interrupção do Consumidor (FIC)

Duração de Interrupção do Consumidor (DIC) 3.2 Índices de Conformidade

Freqüência Equivalente de Violação de Tensão (FEV)

Nível Equivalente de Violação de Tensão (NEV)

Variação Equivalente de Violação de Tensão (VEV)

Duração Equivalente de Violação de Tensão (DEV)

Duração Relativa da Transgressão de Tensão

Precária(DRP)

Duração Relativa da Transgressão de Tensão Crítica (DRC)

Índice de Unidades Consumidoras com Tensão

Crítica(ICC)

4- Variações Momentâneas de Tensão (VMT)

5- Níveis de Severidade de cintilação (Pst, Plt)

6- Desequilíbrio de Tensão (K)

7- Distorção Harmônica total (DHT)

\section{Procedimentos e roteiros metodológicos}

A pesquisa para referendar a metodologia foi realizada por meio da captação dos custos diretos, levando-se em consideração os diversos custos fixos e variáveis devido às interrupções, definidas anteriormente. As pesquisas de campo foram realizados em 17 (dezessete) empresas industriais dos Estados de PE, PB, AL, SE e BA, situadas nos distritos industriais e áreas metropolitanas de João Pessoa, Campina Grande, Recife, Maceió, Aracaju e Salvador .

Os consumidores industriais foram estratificados em diversas variáveis de análise como: 1-Classificação Nacio- nal de Atividade Econômica (CNAE); Porte; Consumo de Energia; Localização Geográfica; Custo da Produção associados às falhas; Número de Empregados; Tempo da Interrupção; Receita Líquida Anual e Lucro Líquido Anual.

A Classificação Nacional de Atividades Econômica (CNAE) define os seguintes ramos de produção: 1-Transformação de Minerais não Metálicos.; 2-Química; 3Metalúrgica; 4-Produtos Farmacêuticos e Veterinários; 5Mecânica; 6-Perfumes, Sabões e Velas; 7-Material Elétrico e de Comunicações; 8-Produtos de Matérias Plásticas; 9-Material de Transporte; 10-Têxtil; 11-Madeira; 12-Vestuário, Calçados e Artigos de Tecidos; 13-Mobiliário; 14Produtos Alimentares; 15-Papel e Papelão; 16-Bebidas; 17-Borracha; 18-Editorial e Gráfica; 19-Couros Peles e Similares.

Quanto aos custos associados às falhas eles foram definidos como:

... Custos de Reparos (CR): Custos relativos aos reparos ou à compra de novos equipamentos danificados pela perda da qualidade de energia elétrica.

... Custos de Produto sem Elaboração Estragados (CEE): Custo direto associado à perda de material durante o processo de manufatura.

... Custos de ProdutosAcabados Estragados(CAE): Custo direto associado à perda de material já fabricado ou em estoque.

... Custo de Matéria Prima ou Produtos Primários Deteriorados (CMP): Custo direto associado à perda de matérias primas ou produtos primários, estocados.

... Custos de Vendas não Realizadas (CV): Custo associado à perda de vendas que não foram feitas devido à interrupção da energia elétrica.

... Custo da Proteção (CPR): Este custo se refere ao gasto que o consumidor industrial tem para se proteger da perda da qualidade de energia elétrica.

... Custo de Geração Própria (CGP): Custo devido à geração de energia alternativa através de geradores de emergência, baterias etc. em caso de interrupção.

... Custo de Perdas de Informações (CIN): Custo associado a perdas de informações guardadas em meio computadorizado decorrente de interrupção ocorrida. Esse custo é estimado pelo cálculo da reposição da informação.

... Custo de Horas Extras (CHE): Custo associado à operação do estabelecimento, quando o seu horário normal de funcionamento é prolongado devido à falha da energia elétrica.

... Custo de Retomada ou Reinício da Produção 
(CRP): Este custo refere-se aos gastos utilizados para se retomar o ritmo normal da produção no caso de ocorrência de uma interrupção. Inclui-se, neste item, os custos de preparo das máquinas, limpeza de resíduos, reposição de ferramentas, reprogramação da produção, reaferição dos equipamentos computadorizados e estabelecimentos dos novos padrões.

Os resultados e os dados coletados foram armazenados num banco de dados onde podem ser calculados alguns indicadores econômicos. Os dados de saídas ou relatórios podem ser expressos em diversas formas como:

1-Por evento, ou seja, por interrupção, "conformidade"ou

$$
I c_{\text {total }} @ \underset{\mathrm{i} @ 1}{\hat{\mathrm{n}}} I c_{i}
$$

flutuação (Unidade $=\mathrm{R} \$$ )

Onde: $I c_{i}$ seria os diversos custos parciais associados definidos:

ICtotal @ $\hat{\underline{I}} I c_{i}=$ Custo de Reparos + Custos de Produtos Produtos Acabados Estragados + Custo de Matéria Prima ou Produtos Primários Deteriorados + Custos de Vendas não Realizadas + Custo da Proteção + Custo de Geração Própria + Custo de Perdas de Informações + Custo de Horas Extras + Custo de Retomada ou Reinício da Produção.

2- Por variável tempo de uma determinada Empresa, por exemplo: Custo de Interrupção (R\$)- 1 segundo, 1 min, e uma hora.

3- Classificação Nacional de Atividade Econômica: Ex: Química, Bebidas, Metalúrgica, Têxtil e outros.

4- Custo das Perdas por Consumo de Energia: (unidade: $\mathrm{R} \$ \mathrm{MWh})$

5- Por Localização Geográfica: Ex: Área do Distrito Industrial do Cabo (Recife), Camaçari, Maceió, João Pessoa ou outra micro área.

6- Por Porte da Indústria: Quantificação pela Receita Líquida.

7- Por Consumo de Energia na Indústria.

\section{Apresentação e discussão dos resultados}

Nas Figuras 4 a 6 são apresentados os resultados dos dados coletados das empresas industriais para os diversos segmentos de atividades econômicas.

Analisando-se os dados das Figuras 4, 5 e 6 têm-se os seguintes comentários:
Os custos das perdas por interrupção para as Empresas 2 e 4 estão na faixa de $\mathrm{R} \$ 270$ mil a R \$ 500 mil. Esses valores estão próximos dos valores médios obtidos que se situam na faixa de R \$ 90mil a R \$ 500mil, conforme se pode observar na curva dos dados médios consolidados no item a seguir e apresentada na Fig 20.

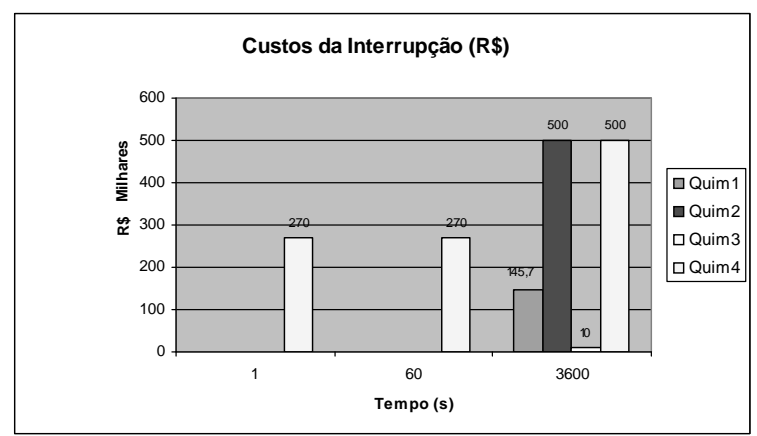

Figura 4 - Custo das Perdas por Interrupção das Empresas Químicas em Função do Tempo.

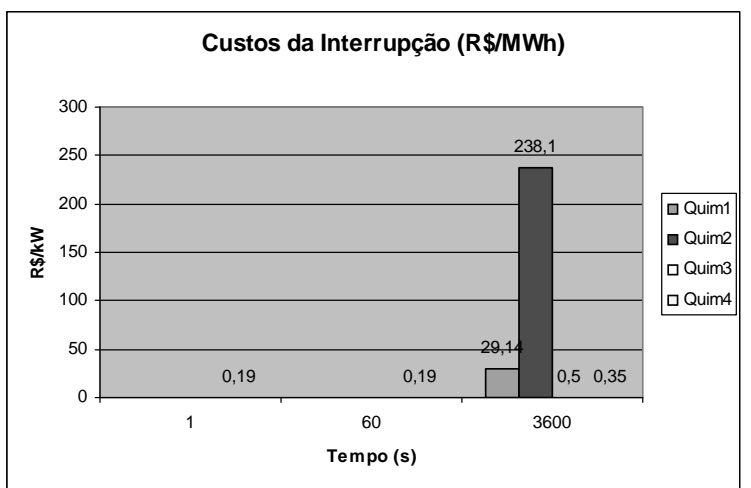

Figura 5 - Custo das Perdas por Interrupção em Relação ao Consumo de Energia (R\$/MWh) das Empresas Químicas em Função do Tempo

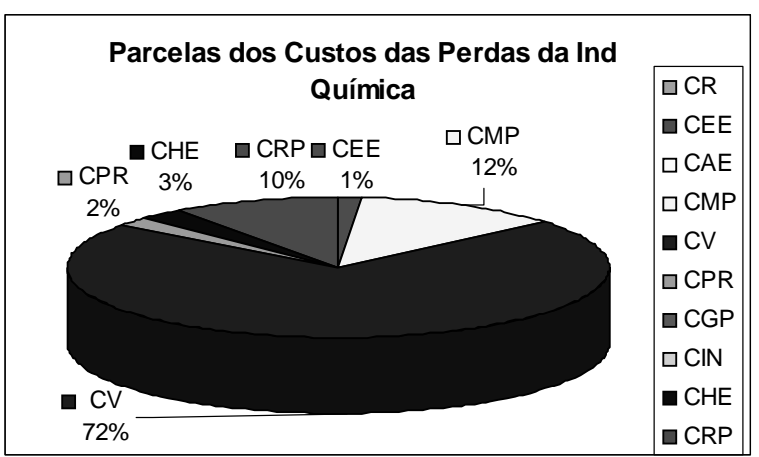

Figura 6 - Gráfico do Detalhamento dos Dados Estimados dos Custos das Perdas da Empresa Química

... Observa-se que o mesmo não acontece com os dados das Empresas 1 e 3 cujos valores são 145 mil e 10 mil bem inferiores aos valores médios encontrados para interrupção de uma hora 500 mil. A Empresa 4, devido a suas características de processos, apesar de ter um consumo de energia (1.428.000 MWh) bem superior ao da 
Empresa 2 (2.100 MWh), tem o mesmo custo de perdas, R\$ 500 mil, para interrupção de uma hora.

... No que refere aos custos por consumo de energia

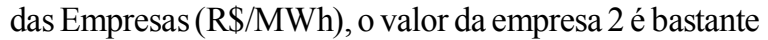
elevado atingindo o valor de 238,10 R\$/MWh, acima da média que é entre 1,5 R \$/MWh e 45 R \$MWh, segundo a consolidação dos resultados apresentado na curva da Figura 21. Nas Empresas 3 e 4, os valores são baixos situados na faixa de 0,3 a $0,5 \mathrm{R} \$ / \mathrm{MWh}$.

... Quanto ao detalhamento dos custos da Indústria Química observa-se que os maiores custos referem-se à parcela Custo de Vendas não Realizadas $(\mathrm{CV})$ responsável por quase $2 / 3$ das perdas, $72 \%$, seguido pelo Custo de Matéria Prima ou Produtos Primários Deteriorados (CMP), 12\%, e o Custo de Retomada da Produção (CRP) $10 \%$.

No que se refere ao segmento das Empresas Metalúrgicas as Figuras 7, 8 e 9 apresentam os dados consolidados de custos das perdas devido à interrupção da energia elétrica.

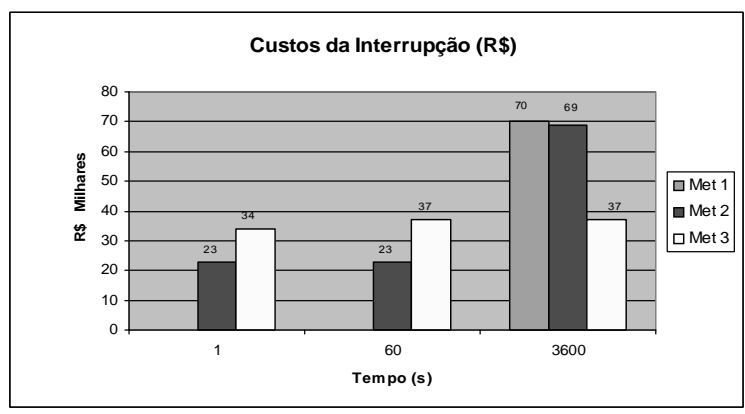

Figura 7 - Custo das Perdas por Interrupção da Empresas Metalúrgicas em função do Tempo

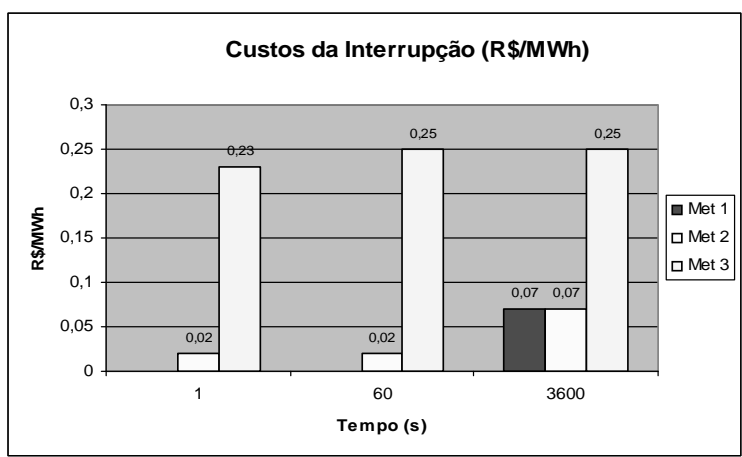

Figura 8. Custo das Perdas por Interrupção em Relação ao Consumo de Energia (R\$/MWh) das Empresas Metalúrgicas em Função do Tempo

Pelos dados das Figuras 7 e 8 apresentados têm-se as seguintes observações:

Os custos das perdas no tempo de $1 \mathrm{~s}$ o valor médio é de R\$30 mil exceto a da Empresa 1, que devido ao seu processo, praticamente não é afetada nesse intervalo de tempo. Com relação às perdas em uma hora o valor fixou-se no patamar de R 70 mil, com exceção da Empresa 3 que é de R $\$ 37$ mil. Esses dados são cerca de $20 \%$ abaixo do valor médio que é $\mathrm{R} \$ 500$ mil.

\begin{tabular}{|c|c|}
\hline $\begin{array}{l}\text { Parcelas dos Custos das Perdas da Ind } \\
\text { Metalúrgica } \\
\square \mathrm{CPR} O \mathrm{CRP} \text { CHE } \\
\mathrm{CV} \\
71 \%\end{array}$ & $\begin{array}{l}\square \mathrm{CR} \\
\square \mathrm{CEE} \\
\square \mathrm{CAE} \\
\square \mathrm{CMP} \\
\square \mathrm{CV} \\
\square \mathrm{CPR} \\
\square \mathrm{CGP} \\
\square \mathrm{CIN} \\
\square \mathrm{CHE} \\
\square \mathrm{CRP}\end{array}$ \\
\hline
\end{tabular}

Figura 9 - Gráfico do Detalhamento dos Dados Estimados dos Custos das Perdas da Empresa Metalúrgica

... No que refere aos custos por consumo de energia (R\$/MWh) os valores das Empresas 1 e 2, são próximos, variando de 0,02 R\$/MWh a 0,07 R \$/MWh. Na Empresa 3 os valores são um pouco maiores alcançando o patamar de 0,25 R\$/MWh. Cabe ressaltar que esses dados estão situados bem abaixo da média que é entre 1,5 R\$/MWh a $45 \mathrm{R} \$ / \mathrm{MWh}$.

... Quanto ao detalhamento dos custos das perdas da Indústria Metalúrgica da Figura 9 observa-se que as parcelas de maiores porcentuais são: Custos de Produtos Acabados Estragados (CAE) 20\%, Custo de Vendas Não realizadas (CV) 70\%, e Custos da Proteção (CPR) 9\%.

Nas Figuras 10 e 11 são apresentados os gráficos dos custos das empresas têxteis.

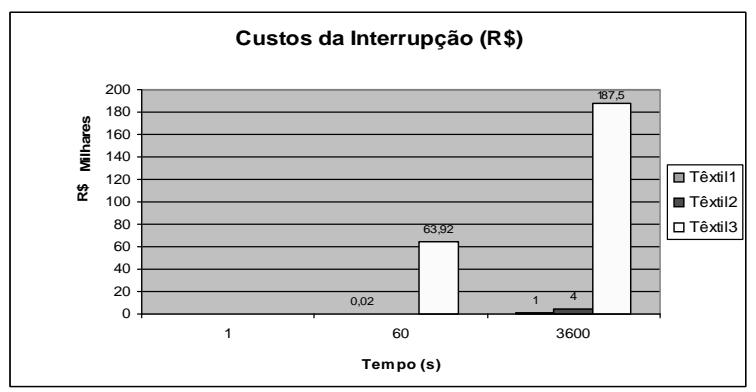

Figura 10 - Custo das Perdas por Interrupção das Empresas Têxteis

Analisando-se os dados, têm-se os seguintes comentários:

... $\quad$ Nas empresas têxteis, os custos das perdas devido à interrupção da energia elétrica durante uma hora nas Empresas 1 e 2 são R \$ 1 mil e R \$ 4 mil, respectivamente. No caso da Empresa 3, os valores se situam na faixa de R $\$ 63$ 
mil a R\$ 187 mil. O consumo de energia na Empresa 1 é $15 \%$ comparado com o da Empresa 2, e o seu faturamento pela receita líquida é de cerca de $3 \%$ da Empresa 2 . Neste caso, observa-se que há uma diferença entre o porte das Empresas, ou seja, a Empresa 1 pode ser considerada uma média empresa e as Empresas 2 e 3 de grande porte, segundo o critério de receita líquida.

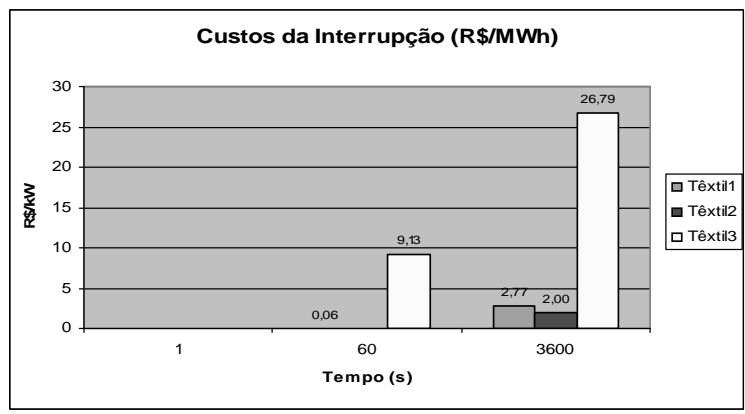

Figura 11 - Custo das Perdas por Interrupção em Relação ao Consumo de Energia (R\$/MWh) das Empresas Têxteis em Função do Tempo

... Entretanto, ao se fazer a análise do custo por consumo de energia em (R\$/MWh), essa grande diferença entre a 1 e a 2 muda, e os valores se aproximam e alcançam os valores para as Empresas 1 e 2 entre 2,00 R\$/MWh e 2,77 R \$/MWh, bem abaixo da média que é $45 \mathrm{R} \$ / \mathrm{MWh}$. No caso da Empresa 3, os valores são mais próximos dos valores médios alcançando o patamar de 26,79 R \$/MWh.

... Quanto ao detalhamento dos custos das perdas na Indústria Têxtil as parcelas mais significativas são: Custos de Reparos (CR) 59\%, Custos da Proteção (CPR) $19 \%$ e, $7 \%$ e Custo de Vendas Não realizadas (CV) 8\%.

Os dados coletados para as Empresas de Produtos Alimentares são apresentados nas Figuras 12 e 13 e na Figura 14 o detalhamento das parcelas que compõe esses custos para uma hora de interrupção.

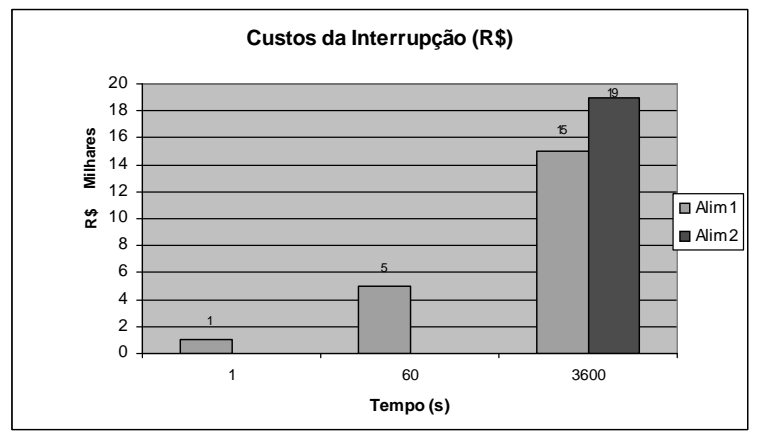

Figura 12 - Custo das Perdas por Interrupção das Empresas de Produtos Alimentares em função do Tempo

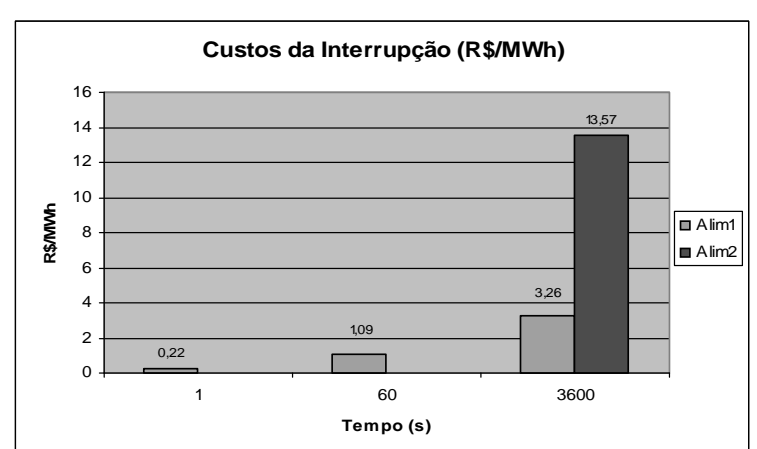

Figura 13 - Custo das Perdas por Interrupção em Relação ao Consumo de Energia (R\$/MWh) das Empresas de Produtos Alimentares em Função do Tempo

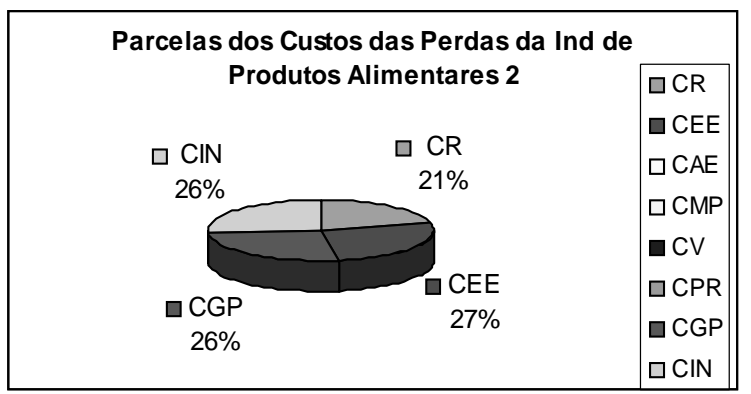

Figura 14 - Gráfico do Detalhamento dos Dados Estimados dos Custos das Perdas da Empresa de Produtos Alimentares

... Pelas observações nas figuras apresentadas verifica-se que os custos das perdas devido à interrupção de energia elétrica deste segmento industrial são considerados bem baixos comparadas com outros. Apesar de serem empresas de médio porte, no tempo de 1 hora, o valor médio é de R\$ 17 mil, muito inferior aos valores médios obtidos em outras empresas industriais que são em torno de R \$ 500 mil algumas alcançando até R \$ 900 mil.

... $\quad$ No que refere aos custos por consumo de energia da empresa (R\$/MWh) observa-se uma variação entre $1 \mathrm{~s}$ e uma hora de 0,2 R\$/MWh a 3,26 R\$/MWh na Empresa 1, ou seja, os valores se situam bem abaixo da média observada que é entre 1,5 R \$/MWh a $45 \mathrm{R} \$ / \mathrm{MWh}$. Na Empresa 2, os dados atingiram o patamar de 13,5 R\$/MWh para uma hora de interrupção, entretanto ainda abaixo do valor médio.

... $\quad$ Na Figura 14, são apresentados o detalhamento dos custos, onde se verifica que a composição foi dos seguintes parcelas: Custos de Produtos em Elaboração Estragados (CEE), Custo de Matéria Prima ou Produtos Primários Deteriorados (CMP), Custo de Horas Extras (CHE) e Custo de Retomada ou Reinício da Produção (CRP). Onde cada custo contribuiu com aproximadamente $1 / 4(25 \%)$ do custo total. 
Nos Gráficos das Figuras 15, 16 e 17 são apresentados os custos das perdas devido à interrupção para as Empresas de Produtos Farmacêuticos e Veterinários e Mecânica.

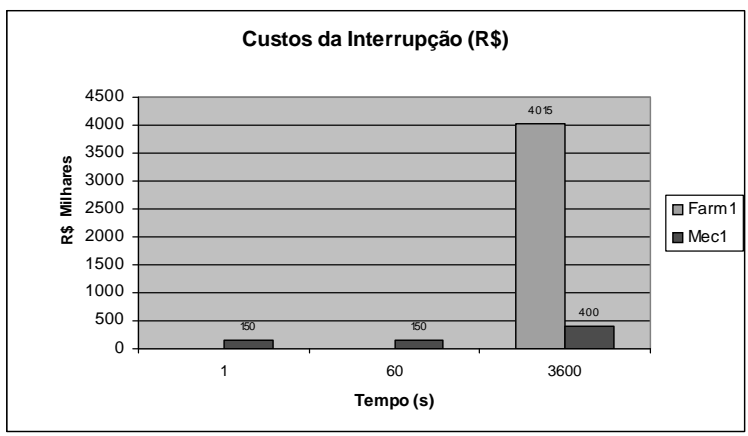

Figura 15 - Custo das Perdas por Interrupção das Empresas de Produtos Farmacêuticos e Veterinários, e Mecânica em função do Tempo

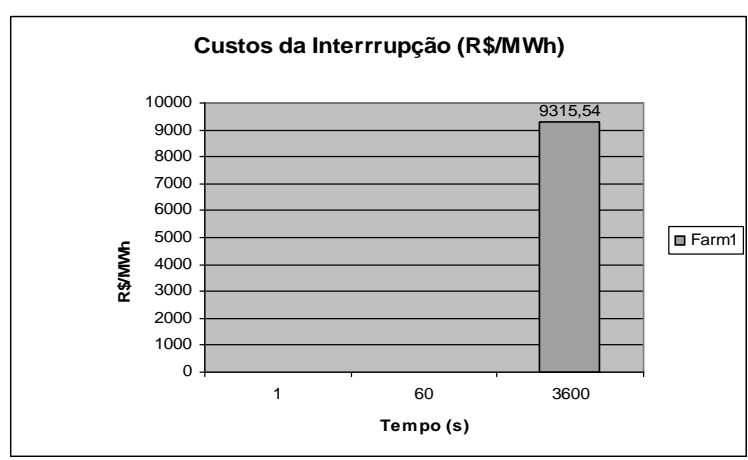

Figura 16 - Custo das Perdas por Interrupção em Relação ao Consumo de Energia (R $\$$ MWh) da Empresa de Produtos Farmacêuticos e Veterinários em função do Tempo

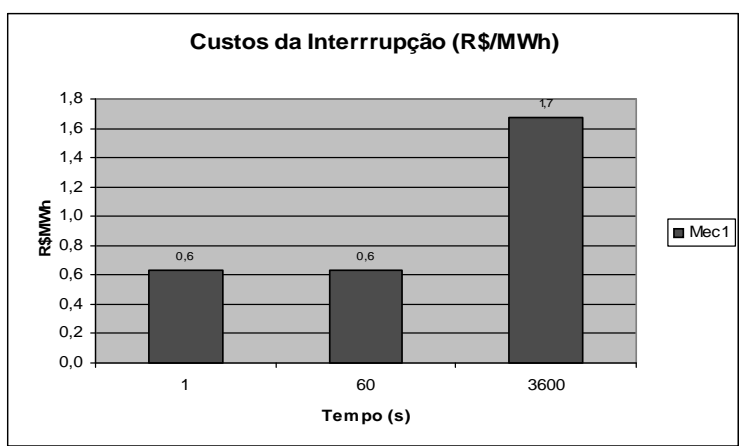

Figura 17 - Custo das Perdas por Interrupção em Relação ao Consumo de Energia (R\$/MWh) da Empresa Mecânica em função do tempo

Analisando-se os dados apresentados, têm-se seguintes observações:

... Esses segmentos industriais têm muitas diferenças entre si, ou seja apesar de empresa do setor de Produ- tos Farmacêuticos e Veterinários ser uma média empresa, os prejuízos são bastante altos superando o valor de $\mathrm{R} \$ 4$ milhões, muito superior à média obtida em outros segmentos industriais que são em torno de R $\$ 90$ mil a $\mathrm{R} \$ 500$ mil. Ressalta-se o alto valor agregado devido à inovação ao produto final deste segmento.

... No que refere aos custos por consumo de energia (R\$/MWh) os valores da Empresa de Produtos Farmacêuticos e Veterinários atingiram o valor de 9.315,54 R\$/ MWh para uma hora, bem superior à média que é $45 \mathrm{R} \$$ / MWh. Este valor é extremamente alto, o que mostra a necessidade de se ter um gerenciamento especial e personalizado para esse segmento industrial.

... Na Empresa Mecânica, os dados atingiram os valores de R \$ 150 mil para 1 segundo até R \$ 400 mil para uma hora, valor que está dentro da média que é de $\mathrm{R} \$ 90$ mil a $\mathrm{R} \$ 500$ mil.

.. $\quad$ No que refere aos custos das perdas por consumo de energia (R\$/MWh), os valores da Empresa Mecânica variam de 0,6 R\$/MWh, para um segundo, até 1,67 R\$/ MWh para a interrupção de uma hora, bem abaixo dos valores médios de $45 \mathrm{R} \$ / \mathrm{MWh}$.

... No caso da composição de custos para o setor de Produtos Farmacêuticos e Veterinários, os valores mais significativos foram o Custo dos Reparos (CR) 31\%, Custos de Vendas não Realizadas (CV) $15 \%$ e Custo de Perdas de Informações (CIN) 30\%, ou seja estas parcelas representam cerca de $3 / 4$ (75\%) dos Custos das Perdas Totais.

Os dados coletados para as Empresas de Transformação Minerais não Metálicos, Materiais Elétricos e de Comunicação e de Vestuário, Calçados e Art. de Tecidos são mostrados a seguir (FIGURA 18 e 19).

Figura 18. Custo das Perdas por Interrupção das Empresas de

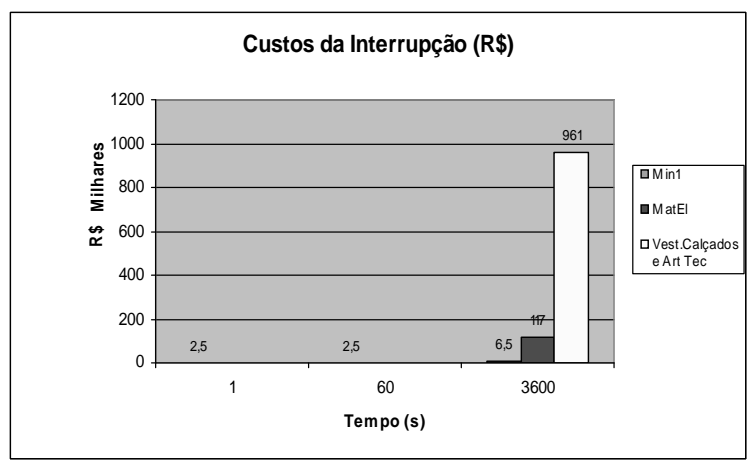

Transf. Minerais não Metálico, Mat. Elétricos e de Com. e de Vest., Calçados e Art. de Tecidos

Analisando-se os dados apresentados, têm-se seguintes observações: 


\section{... Na Empresa de Transformação de Minerais Não} Metálicos, os valores são extremamente baixos alcançando R \$ 2,5 mil para interrupções de um segundo até o patamar de R \$ 6,5 mil muito inferior aos valores médios. No segmento da Empresa de Materiais Elétricos e de Comunicações, os valores alcançam R \$ 117 mil para a interrupção de uma hora.

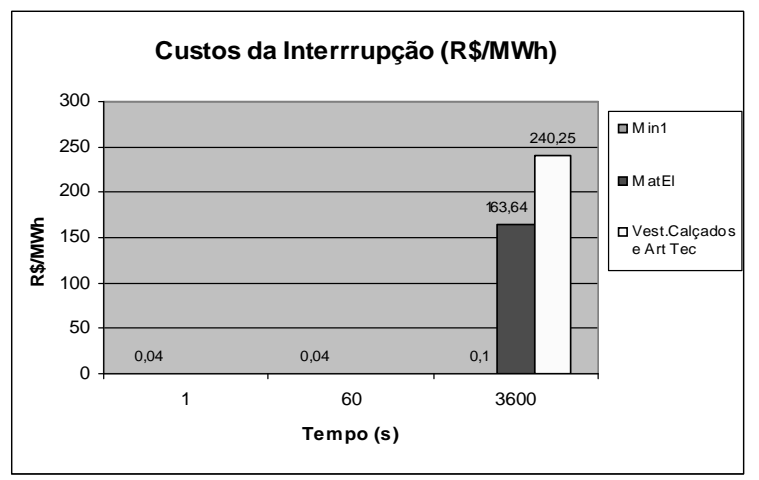

Figura 19 - Custo das Perdas de Int.em Relação ao Consumo de Energia (R\$/MWh) das Emp. Transf. Min. não Met., Mat. Elét.e de Com. e de Vest., Calçados e Art. Tecidos

.. $\quad$ Na empresa de Vestuário, Calçados e Artigos de Tecidos, o custo da interrupção de energia em uma hora atinge o valor de $\mathrm{R} \$ 961$ mil, acima do valor médio de $\mathrm{R} \$$ 500 mil para a interrupção de uma hora.

... No que refere aos custos das perdas por consumo de energia (R\$/MWh), o segmento de Minerais não Metálicos os valores são extremamente baixos situando-se na faixa de $0,1 \mathrm{R} \$ / \mathrm{MWh}$, bem abaixo da média.

... Nos segmentos industriais de Empresa de Materiais Elétricos e de Comunicações e Vestuário, Calçados e Artigos de Tecidos, os valores alcançam 163,64 R\$/MWh e 240,25 R\$/MWh, respectivamente. Esses dados são bem superiores ao valor médio de 45 R\$/MWh.

... $\quad$ No que se refere às parcelas que compõem para o setor de Materiais Elétricos e de Comunicações, os custos são: Custo dos Reparos (CR) 31\%, Custo de Vendas não Realizadas (CV) 15\% e Custo de Perdas de Informações (CIN) 30\%, ou seja, estas parcelas representam cerca de $3 / 4(75 \%)$ dos Custos das Perdas Totais.

... Quanto àcomposição dos custos da empresa do setor de Vestuário, Calçados e Artigos de Tecidos, observa-se que as parcelas mais significativas que compõem os custos são: Custo dos Produtos em Elaboração Estragados (CEE) 38\%, o Custo de Vendas não Realizadas (CV) $36 \%$ e o Custo de Produtos Acabados Estragados (CAE) $16 \%$, parcelas que correspondem a $90 \%$ dos Custos das Perdas Totais.

\subsection{Consolidação dos resultados de custos das perdas}

Analisando-se, de forma geral, os custos de todas as empresas analisadas foi plotado um gráfico com a equação básica que representasse estes custos. Esta estimativa foi feita tanto para os custos totais ( $R \$$ ) como para os custos por consumo de energia (R\$/ MWh). Nas Figuras 20 e 21 são apresentadas estes gráficos dos Custos de Perdas da Interrupção.

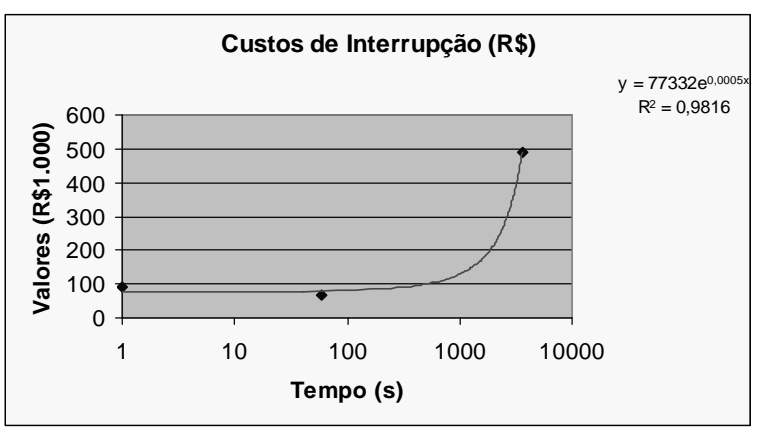

Figura 20 - Custo das Perdas por Interrupção de Energia Elétrica das Empresas em Função do Tempo de Interrupção em segundos

Verifica-se, na Figura 20 que os valores médios dos custos em função do tempo de interrupção situaram-se na faixa de R $\$ 90$ mil de 1 segundo, até aproximadamente 2 minutos (120 segundos) e em seguida há um crescimento até atingir o valor de $\mathrm{R} \$ 500$ mil para uma hora (3600 segundos). A equação da curva encontrada foi: $y @ 77.332 e^{0,00005 x}$.

Quanto aos Custos das Perdas por Interrupção de Energia Elétrica em Relação ao Consumo de Energia em Função do Tempo (em segundos), conforme é apresentado na Figura 21, os valores médios ficaram no patamar de 1,5 R \$/MWh para a interrupção de um segundo até em torno de 2 minutos (120 segundos) para em seguida alcançar o valor de $45 \mathrm{R}$ /MWh em uma hora (3600 segundos). A equação da curva encontrada foi: $y @ 10,302 e^{0,0003 x}$

Na Figura 22 é apresentado o gráfico das curvas de Custos Médios em função do tempo de interrupção calculados na Empresa Mecânica e de Produtos Alimentares. Verifica-se que os valores médios dos custos em função do tempo de interrupção da empresa Mecânica que atinge o valor entre $\mathrm{R} \$ 150.000$ a R \$ 400.000 é bem superior ao da Empresa de Produtos Alimentares que se situa na faixa de R\$17.000. 


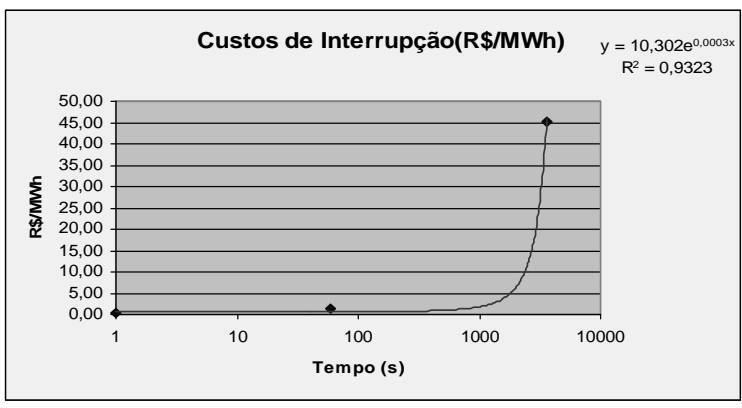

Figura 21 - Custo das Perdas por Interrupção de Energia Elétrica em Relação ao Potência Instalada das Empresas em Função do Tempo de Interrupção (R\$/MWh)

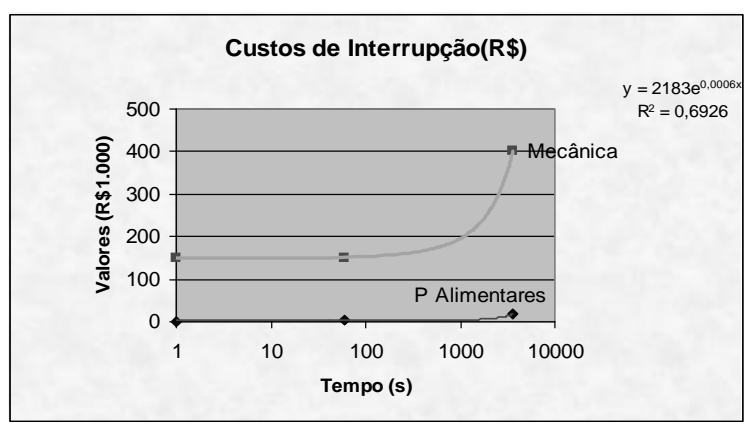

Figura 22 - Custo das Perdas por Interrupção de Energia Elétrica das Empresas Mecânica e de Produtos Alimentares em Função do Tempo de Interrupção

Quanto aos Custos das Perdas por Consumo de Energia (R\$/MWh), apresentado na Figura 23, a situação inverteu-se e os valores médios da Empresa de Produtos Alimentares atingiu o patamar de 8,4 R \$/MWh bem superior ao da Empresa Mecânica cujo valor foi 1,7 R\$/MWh.

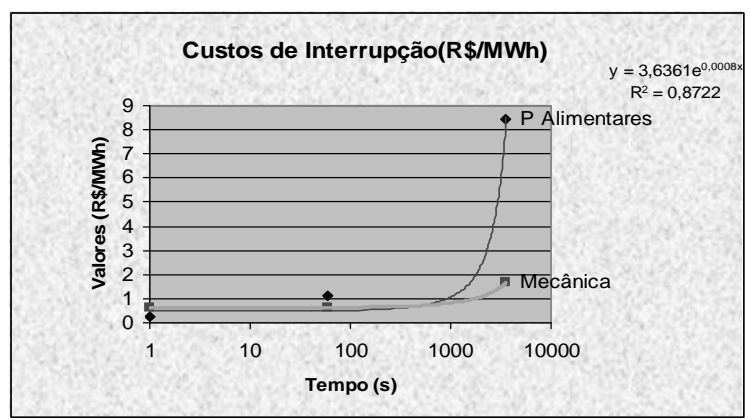

Figura 23. Custo das Perdas por Interrupção de Energia Elétrica em Relação à Potência Instalada das Empresas em Função do Tempo de Interrupção (R\$/MWh)

\section{Conclusões}

Observa-se que a oferta de energia elétrica tem uma relação direta com o crescimento econômico do País. O esforço concentrado no setor elétrico ao longo das últimas décadas foi decisivo para a Região, e será o responsável nas próximas décadas cada vez mais pelo papel de princi- pal indutor do desenvolvimento econômico da Região Nordeste. Ter uma boa qualidade de energia elétrica, com menores custos e mais eficiente é fundamental para se obter uma melhor competitividade dos produtos industriais e conseqüentemente uma contribuição para o crescimento deste setor.

A metodologia tradicional com os seus indicadores "Elétricos" necessita ser aperfeiçoada e os dados são insuficientes para uma tomada de decisão na gestão da energia elétrica. Com a introdução dessa nova "Proposta de Metodologia de Avaliação da Qualidade de Energia Elétrica na Produção Industrial" haverá subsídios para uma melhoria na gestão da operação e na avaliação do fornecimento de energia elétrica aos consumidores industriais.

Esta nova abordagem fornecerá dados principalmente de custos das perdas que prejuízos adicionais industriais associados à perda desta qualidade de energia elétrica fornecendo informações para o planejamento da expansão das empresas de energia elétrica, e também para os órgãos reguladores e Federação das Indústrias.

Esta metodologia também seria de grande utilidade para o sistema de gestão do planejamento estratégico das empresas de energia e industriais, onde se poderiam selecionar e implantar áreas com um alto nível de qualidade de energia. Nesses locais denominados "Ilhas de Qualidade de Energia Elétrica", poderiam ser instaladas empresas industriais de alta tecnologia muito sensíveis à energia elétrica e alto valor agregado aos seus produtos.

Haverá também uma contribuição efetiva para o planejamento da manutenção da empresa distribuidora e transmissora de energia elétrica. Poderia inclusive, instalar equipamentos especializados que contribuam para uma melhor confiabilidade do fornecimento de energia elétrica na área industrial.

\section{REFERÊNCIAS}

ALVES, M.; RIBEIRO, T. Análise da Qualidade de Energia Elétrica: Metodologia e Caso Exemplo. In: Anais III SBQEE - Seminário Brasileiro sobre Qualidade de Energia Elétrica, ABrasília, 1999.

AMANTEGUI, J. et al. Characterization of Voltage Dips in Electrical Networks And Their Impact on Customer Installations. In: Proceedings International Conference on Large Electric Network CIGRÉ,Paris, 1998.

BILLINTON, R.; WACKER, G. Customer Costs of Electric Service Interruptions, Proceedings of the IEEE, vol. 77, no. 6, June 1989, p. 919-930.

BOLLEN, M.; YALÇINKAYA, G.; HAZZA, G. The Use of 
Electromagnetic Transient Programs for Voltage Sag Analysis. In: Proceedings 8th International Conference on Harmonics and Quality of Power, Athens-Greece, Oct. 1998.

BONATTO, B.; MERTEUS, E.; FERNANDES, F. Diagnóstico da Qualidade de Energia Elétrica em Clientes Industriais. In: Anais III SBQEE - Seminário Brasileiro sobre a Qualidade de Energia Elétrica, Brasília, 1999.

BRONZEADO, H. et al. Uma Proposta de Nomenclatura Nacional de Termos e Definições Associados à Qualidade de Energia Elétrica. In: Anais II SBQEE-Seminário Brasileiro da Qualidade da Energia Elétrica, Itajubá, Brasil, Nov. 1997.

CAVALCANTI, Guilherme A. O Nordeste do Brasil: Políticas Energéticas e Desenvolvimento Regional. Grenoble, França, 1985: Universidade de Ciências Sociais de Grenoble, (Tese, Doutorado em Economia).

CAVALCANTI, Roberto. Fundamentos de uma Estratégia de Desenvolvimento do Nordeste. In: Anais Fórum Nacional, Seminário Especial 50 Anos de Desenvolvimento: O Papel do BNDES e do Banco do Nordeste - 1952/2002, Rio de Janeiro, Set. 2002.

CHESF. Companhia Hidroelétrica do São Francisco-Indicadores. Disponível em: < http://www.chesf.gov.br/xml/ energia_indicadores.php $>$. Acesso em: Jan. 2006.

DIALYNAS, E.; KOSKOLOS N.; MEGALOCOMOS, S. Assessment of Power Interruption Costs for the Industrial Customers in Greece. In: Proceedings 8th International Conference on Harmonics and Quality of Power, AthensGreece, Oct. 1998.

GARCIA, F.; PEEL, M.; GURLASKIE, G. Aplicação de Equipamentos à Base de Eletrônica de Potência para a Melhoria da Qualidade de Energia de Sistemas de Distribuição: Estudo de Casos. In: Anais III SBQEE-Seminário Brasileiro sobre Qualidade de Energia Elétrica, Brasília,

GOVERNO DE SÂO PAULO. Qualidade do Fornecimento de Energia Elétrica: Indicadores, Padrões e Penalidades-Versão 2, Secretaria de Energia Comissão de Serviços Públicos de Energia, São Paulo: Governo São Paulo, Jul. 1997.

HEYDT, G., JEWELL, W. Pitfalls of Electric Power Quality Indices, IEEE Transactions on Power Delivery, v. 13, no. 2, p. 570-578,Apr. 1998.

IBGE. Instituto Brasileiro de Geografia e Estatística. Contas Nacionais do Brasil $2003 \mathrm{n}^{\circ}$. 15-Contas Regionais do Brasil 2003, Rio de Janeiro, 2005.
IEEE-PESPOWER ENGINEERING SOCIETY.IEEE Guide for Service to Equipment Sensitive to Momentary Voltage Disturbances, USA: IEEE, June 1995.

IEEE-PES POWER ENGINEERING SOCIETY. IEEE Recommended Practice for Monitoring Electric Power Quality, USA: IEEE, Nov. 1995.

KARIUKI, K.;ALLAN, R. Evaluation of Reliability Worth and Value of Lost Load. IEEE Proceedings - General Transmission Distribution, vol. 143, n. 2,p. 171-180, 1996.

LEITÃO, José Júlio A. Impactos Econômicos Causados pelos Distúrbios na Rede Básica de Energia Elétrica. São Paulo, 2002: Universidade de São Paulo, (Tese, Doutorado em Engenharia).

MAGALHÃES, Cecília et al. Custo da Interrupção do Fornecimento de Energia Elétrica. In: Anais IV SBQEE Seminário Brasileiro sobre Qualidade de Energia Elétrica, Porto Alegre, Brasil, Ag. 2001.

MCGRANAGHAN, M.; ROETTGER B. Economic Evaluation of Power Quality, IEEE Power Engineering Review, USA, Feb. 2002.

OLESKOVICZ, Mário. Qualidade da Energia - Fundamentos Básicos. Disponível em: <http:// www.sel.eesc.sc.usp.br/pos/disciplinas/se15749/ Apost_QEE_2004.pdf>.Acesso em: Jan. 2006.

PEREIRA, F. et al. An Analysis of Costs Related to the Loss of Power Quality. In: Proceedings 8th International Conference on Harmonics and Quality of Power, AthensGreece, Oct. 1998.

STECIUK, P.; REDMON, J. Voltage Sag Analysis Peaks Customer Service. IEEE Computer Applications in Power, p. 48-51, 1996.

SULLIVAN, M.J. et al. An Interruption Costs, Customer Satisfaction and Expectations for service Reliability. IEEE Transactions on Power Systems, v. 11, n. 2, p. 989-995, 1996. 